\title{
Global entanglement and coherent states in an $N$-partite system
}

\author{
Piroska Dömötör* and Mihály G. Benedict ${ }^{\dagger}$ \\ Department of Theoretical Physics, University of Szeged, Hungary
}

\begin{abstract}
We consider a quantum system consisting of N parts, each of which is a "quKit" described by a $K$ dimensional Hilbert space. We prove that in the symmetric subspace, $\mathbb{S}$, a pure state is not globally entangled, if and only if it is a coherent state. It is also shown that in the orthogonal complement $\mathbb{S}_{\perp}$ all states are globally entangled.
\end{abstract}

\section{Introduction}

The property of entanglement of composite systems is considered to be the most pronounced difference between classical and quantum systems, and it has become especially in the center of interest since the realization that it can serve as a resource for quantum computation and communication [1]. The definition of entanglement is easily understood for pure states, while the full characterization of the entanglement properties of mixed states is a difficult and still unsolved mathematical problem [2]. We note, however, that from the point of view of physical applications, pure state entanglement does not seem to be less important, as quantum algorithms and communication protocols are in principle based on pure states [1].

Another important concept widely used in quantum mechanics is the notion of coherent, or quasiclassical states Introduced first for the harmonic oscillator [3] the construction has been extended to several other systems, and the general treatment of coherent states can be found in the books [4, 5, 6]. Oscillator coherent states are exceptional in many respects, they yield the classical results for the expectation values of all the pertinent operators, and they are pointer states for a particle moving in one dimension in a disspative environment [7]. A similar result holds for a collection of spins [8]. From the point of view of the present work we quote the paper [9], where coherent states of several two level atoms (or qubits) are considered, and the review [10], where coherent states are considered in a more general context of Lie groups and algebras. We shall use the methods summarized in that work,

*Domotor.Piroska@stud.u-szeged.hu

†benedict@physx.u-szeged.hu 
but our presentation should be understood in terms of the usual properties of Hilbert space vectors.

In a previous paper [11] we considered the question of entanglement of $N$ qubits, while here we present the extension of the problem to the case of "quKits", where the constituent systems are of arbitrary finite dimension, $K$. We shall prove the statement that a multipartite pure quKit state in the symmetric subspace is not entangled if and only if it is a coherent state, while in the subspace orthogonal to the symmetric one, all states are entangled. A related property between coherent states and entanglement was pointed out earlier in [12] for a two-partite system. We also cite a recent work on symmetric states and entanglement [13].

\section{Multipartite system of quKits, and operators on it}

We consider an $N$ partite system where each constituent is described by a $K$ dimensional Hilbert space, $\mathfrak{H} \equiv \mathbb{C}^{K}(j=1, \ldots N)$ the elements of which can be called quKits. A general pure state in the tensor product $\mathfrak{H}^{\otimes N} \equiv \mathbb{C}^{K^{N}}$ can be given in a product basis $\bigotimes_{j=1}^{N}\left|k_{j}\right\rangle \equiv$ $\left|k_{1}\right\rangle\left|k_{2}\right\rangle \ldots\left|k_{N}\right\rangle$, formed by some fixed orthonormal bases from each subsystem $\{|k\rangle\}=$ $(|1\rangle,|2\rangle, \ldots|K\rangle)$. Then a general pure state in $\mathbb{C}^{K^{N}}$ is

$$
|\psi\rangle=\sum_{k_{1}, k_{1}, k_{2} \ldots k_{N}=1}^{K} c\left(k_{1} k_{2} \ldots k_{N}\right)\left|k_{1}\right\rangle\left|k_{2}\right\rangle \ldots\left|k_{N}\right\rangle
$$

where $c\left(k_{1} k_{2} \ldots k_{N}\right)$ are complex constants indexed by the $K^{N}$ possible values of the set in the parentheses, and normalized to $1: \sum_{k_{1}, k_{1}, k_{2} \ldots k_{N}=1}^{K}\left|c\left(k_{1} k_{2} \ldots k_{N}\right)\right|^{2}=1$. Operators in the tensor product space are built from those in the individual $\mathfrak{H}$-s, and we shall use a standard set of operators (an operator basis) in each subspace. The raising and lowering operators are defined as $E_{j i}^{\dagger}=E_{i j}=|i\rangle\langle j|$ and $E_{j i}=|j\rangle\langle i|$ for all $i>j$, their number is $K(K-1) / 2$. They are traceless operators acting on the chosen basis elements in the following way: $E_{i j}|k\rangle=\delta_{k j}|i\rangle$ and $E_{j i}|k\rangle=\delta_{k i}|j\rangle$. Their commutator is easily found to be $\left[E_{i j}, E_{i j}^{\dagger}\right]=|i\rangle\langle i|-| j\rangle\langle j|=: H_{i j}$ which is also traceless and diagonal in the selected basis. In the $K$ dimensional space there are only $K-1$ linearly independent operators (apart from the identity) that can be diagonalized simultaneously, and we can choose them to be the $H_{i+1, i}$. The operators $E_{i j}, E_{i j}^{\dagger}, H_{i+1, i}$ form a basis, called the Cartan-Weyl basis in the (complex) space of the $K$ dimensional traceless operators, spanning the Lie algebra $\operatorname{sl}(K, \mathbb{C})$ [14]. In the $K=2$ case (qubits) they are equivalent to the Pauli operators: $H_{21} \simeq \sigma_{3}$, $E_{12}^{\dagger}=E_{21} \simeq \sigma^{+}=\left(\sigma_{1}+i \sigma_{2}\right) / 2, E_{12} \simeq \sigma^{-}=\left(\sigma_{1}-i \sigma_{2}\right) / 2$.

The corresponding collective operators in the $\bigotimes_{j=1}^{N} \mathfrak{H}^{j}$ product space are defined as

$$
\tilde{X}=\sum_{n=1}^{N} X^{(n)}, \quad \text { where } \quad X^{(n)}=\underset{1}{\mathbf{1}} \otimes \underset{2}{\mathbf{1}} \otimes \cdots \otimes \underset{n}{X} \otimes \cdots \otimes \underset{N}{\mathbf{1}}
$$


acts nontrivially only on the $n$th subsystem.

\section{A condition of unentanglement}

A pure $N$ partite state is not entangled by definition, if it is a product state. For a system consisting of $N$ identical quKits we shall use a formal inductive method in order to decide if a pure state is entangled or not. This is a generalization of a condition given by Meyer and Wallach [15]

Any pure state $|\psi\rangle \in \mathbb{C}^{K^{N}}$, which is expanded in the standard basis $\{|k\rangle\}^{\otimes N}$, can be decomposed for each $n=1,2 \ldots N$ quKit as

$$
|\psi\rangle=|1\rangle_{n} \otimes\left|u_{n}^{1}\right\rangle+|2\rangle_{n} \otimes\left|u_{n}^{2}\right\rangle+|3\rangle_{n} \otimes\left|u_{n}^{3}\right\rangle+\cdots+|K\rangle_{n} \otimes\left|u_{n}^{K}\right\rangle=\sum_{k=1}^{K}|k\rangle_{n} \otimes\left|u_{n}^{k}\right\rangle
$$

where the kets $|k\rangle_{n}$ are the basis vectors in $\mathfrak{H}^{n}$ and $\left|u_{n}^{k}\right\rangle(k=1, \cdots K)$ are vectors in $\mathbb{C}^{K^{N-1}}$ which are not normalized in general. Using the above decomposition (3), it can be simply proven that $|\psi\rangle$ is a product state $\left(|\psi\rangle\right.$ is not entangled), if and only if the vectors $\left|u_{n}^{k}\right\rangle$ are parallel to each other $\left(\left|u_{n}^{1}\right\rangle \|\left|u_{n}^{2}\right\rangle\|\cdots\|\left|u_{n}^{K}\right\rangle\right)$ for all possible $n$.

In order to see this, first assume that $|\psi\rangle$ is a product state, and therefore it can be written as

$$
|\psi\rangle=\bigotimes_{n=1}^{N}\left(a_{n}^{1}|1\rangle_{n}+a_{n}^{2}|2\rangle_{n}+\ldots a_{n}^{K}|K\rangle_{n}\right)=\bigotimes_{n=1}^{N} \sum_{k=1}^{K} a_{n}^{k}|k\rangle_{n}
$$

with some $a_{n}^{k}$, obeying $\sum_{k=1}^{K}\left|a_{n}^{k}\right|^{2}=1$ for each $n$. We can write this product state as

$$
\begin{aligned}
|\psi\rangle & =a_{n}^{1}|1\rangle_{n} \bigotimes_{j \neq n}^{N} \sum_{k=1}^{K} a_{j}^{k}|k\rangle_{j}+a_{n}^{2}|2\rangle_{n} \bigotimes_{j \neq n}^{N} \sum_{k=1}^{K} a_{j}^{k}|k\rangle_{j} \ldots+a_{n}^{K}|K\rangle_{n} \bigotimes_{j \neq n}^{N} \sum_{k=1}^{K} a_{j}^{k}|k\rangle_{j}= \\
& =\sum_{k=1}^{K}|k\rangle_{n}\left\{a_{n}^{k} \bigotimes_{j \neq n}^{N} \sum_{k=1}^{K} a_{j}^{k}|k\rangle_{j}\right\}
\end{aligned}
$$

for each $n$. We have then $\left|u_{n}^{k}\right\rangle=a_{n}^{k} \bigotimes_{j \neq n}^{N} \sum_{k=1}^{K} a_{j}^{k}|k\rangle_{j}$, which means that all the $\left|u_{n}^{k}\right\rangle$-s are parallel for a fixed $n$ in the decomposition (3).

Consider now the reverse statement and assume parallellity of $\left|u_{n}^{k}\right\rangle$-s for each fixed $n=1,2 \ldots N$ in (3). In other words we assume that $\left|u_{n}^{k}\right\rangle=\beta_{n}^{k}\left|u_{n}^{1}\right\rangle$ for $k=2, \cdots K$ with some coefficients $\beta_{n}^{k} \in \mathbb{C}$.

Then $|\psi\rangle$ can be written in the form:

$$
|\psi\rangle=\left(1+\sum_{k=2}^{K}\left|\beta_{n}^{k}\right|^{2}\right)^{-1 / 2}\left(|1\rangle_{n}+\sum_{k=2}^{K} \beta_{n}^{k}|k\rangle_{n}\right) \otimes\left|\tilde{u}_{n}^{1}\right\rangle \quad \forall n
$$


and we prove by induction that $|\psi\rangle$ is a product state (Here the $N-1$ quKit states $\left|\tilde{u}_{n}^{k}\right\rangle=$ $\left|u_{n}^{k}\right\rangle / \sqrt{\left\langle u_{n}^{k} \mid u_{n}^{k}\right\rangle}$ are normalized.) For $N=2$ this is obviously true, because then $|\psi\rangle=$ $\left(1+\sum_{k=2}^{K}\left|\beta^{k}\right|^{2}\right)^{-1 / 2}\left(|1\rangle_{1}+\sum_{k=2}^{K} \beta_{1}^{k}|k\rangle_{1}\right) \otimes\left|\tilde{u}_{1}^{1}\right\rangle$ and $\left|\tilde{u}_{1}^{1}\right\rangle$ is a one quKit state.

Suppose now that the statement is true for a system of $N-1$ quKits, and proceed to $N$. We first use the decomposition (6) with respect to the $i$-th $q u K i t$, where $\left|\tilde{u}^{i}\right\rangle \in \mathbb{C}^{K^{N-1}}$ is now an $N-1$ quKit state. Decompose $\left|\tilde{u}^{i}\right\rangle$ further, with respect to the $j$-th quKit:

$$
\begin{aligned}
|\psi\rangle & =\frac{\left(|1\rangle_{i}+\sum_{k=2}^{K} \beta_{i}^{k}|k\rangle_{i}\right)}{\sqrt{1+\sum_{k=2}^{K}\left|\beta_{i}^{k}\right|^{2}}} \otimes\left|\tilde{u}_{i}^{1}\right\rangle=\frac{\left(|1\rangle_{i}+\sum_{k=2}^{K} \beta_{i}^{k}|k\rangle_{i}\right)}{\sqrt{1+\sum_{k=2}^{K}\left|\beta_{i}^{k}\right|^{2}}} \otimes\left(\sum_{k=1}^{K}|k\rangle_{j} \otimes\left|u_{i j}^{k}\right\rangle\right) \\
& =\sum_{k=1}^{K}|k\rangle_{j} \otimes\left(\frac{\left(|1\rangle_{i}+\sum_{k=2}^{K} \beta_{i}^{k}|k\rangle_{i}\right)}{\sqrt{1+\sum_{k=2}^{K}\left|\beta_{i}^{k}\right|^{2}}} \otimes\left|u_{i j}^{k}\right\rangle\right)
\end{aligned}
$$

and compare this with

$$
|\psi\rangle=\frac{\left(|1\rangle_{j}+\sum_{k=2}^{K} \beta_{j}^{k}|k\rangle_{j}\right)}{\sqrt{1+\sum_{k=2}^{K}\left|\beta_{j}^{k}\right|^{2}}} \otimes\left|\tilde{u}_{j}^{1}\right\rangle
$$

As a result we get

$$
\begin{aligned}
\frac{\left|\tilde{u}_{j}^{1}\right\rangle}{\sqrt{1+\sum_{k=2}^{K}\left|\beta_{j}^{k}\right|^{2}}}=\frac{\left(|1\rangle_{i}+\sum_{k=2}^{K} \beta_{i}^{k}|k\rangle_{i}\right)}{\sqrt{1+\sum_{k=2}^{K}\left|\beta_{i}^{k}\right|^{2}}} \otimes\left|u_{i j}^{1}\right\rangle \\
\beta_{j}^{k} \frac{\left|\tilde{u}_{j}^{1}\right\rangle}{\sqrt{1+\sum_{k=2}^{K}\left|\beta_{j}^{k}\right|^{2}}}=\frac{\left(|1\rangle_{i}+\sum_{k=2}^{K} \beta_{i}^{k}|k\rangle_{i}\right)}{\sqrt{1+\sum_{k=2}^{K}\left|\beta_{i}^{k}\right|^{2}}} \otimes\left|u_{i j}^{k}\right\rangle \quad \text { for all } k=2, \cdots K
\end{aligned}
$$

which implies that $\beta_{j}^{k}\left|u_{i j}^{1}\right\rangle=\left|u_{i j}^{k}\right\rangle$. As by hypothesis $\left|\tilde{u}_{i}^{1}\right\rangle$ is a product state, and according to $|\psi\rangle=\left(1+\sum_{k=2}^{K}\left|\beta_{i}^{k}\right|^{2}\right)^{-\frac{1}{2}}\left(|1\rangle_{i}+\sum_{k=2}^{K} \beta_{i}^{k}|k\rangle_{i}\right) \otimes\left|\tilde{u}_{i}^{1}\right\rangle$, the $N$ quKit state $|\psi\rangle$ is also a product state.

\section{The symmetric subspace $\mathbb{S}$ of $\mathbb{C}^{K^{N}}$}

We use the standard procedure to construct the symmetric subspace. Consider a state, where the number of subsystems in states $|1\rangle,|2\rangle,|3\rangle ; \cdots|K\rangle$; are $n_{1}, n_{2}, n_{3}, \cdots n_{K}$, respectively, with $n_{k} \in \mathbb{N}$. We have of course $\sum_{k=1}^{K} n_{k}=N$. Starting from a specific nonsymmetric state where we do know which of the subsystems are in the specified states, e.g. from

$$
\left|\varphi\left(n_{1}, \ldots, n_{K}\right)\right\rangle:=\underbrace{|1\rangle|1\rangle \ldots|1\rangle}_{n_{1}} \underbrace{|2\rangle \ldots|2\rangle}_{n_{2}}|3\rangle \ldots \ldots|K-1\rangle \underbrace{|K\rangle \ldots|K\rangle}_{n_{K}}
$$


we get a symmetric state when applying the symmetrizer $\mathcal{S}$ :

$$
\mathcal{S}\left|\varphi\left(n_{1}, \ldots, n_{K}\right)\right\rangle=\mathcal{C} \sum_{\nu} P_{\nu}\left|\varphi\left(n_{1}, \ldots, n_{K}\right)\right\rangle=:\left|n_{1}, n_{2} \ldots n_{K}\right\rangle_{S}
$$

where $P_{\nu}$ runs over all the permutations of the $N$ subsystems, and $\mathcal{C}$ is an appropriate normalization constant. This state also shares the property, that the number of subsystems in the specified state $|k\rangle$ is $n_{k}$, but we do not know which of the subsystems is in a given basis state, as all such possibilities have the same amplitude.

The number of the possible different symmetric states is easily obtained to be

$$
\left(\begin{array}{c}
N+K-1 \\
N
\end{array}\right)=\operatorname{dim} \mathbb{S}
$$

as it is well known.

Alternatively we can get these symmetric states by applying raising operators to the specific state $\left|\varphi\left(n_{1}=N, n_{2}=0, \ldots, n_{K}=0\right)\right\rangle=|\varphi(N, 0, \ldots, 0)\rangle=|1\rangle_{1} \otimes|1\rangle_{2} \otimes \cdots \otimes|1\rangle_{N} \equiv$ $|1,1 \cdots 1\rangle$, which is obviously symmetric. The state $|1,1 \cdots, 1\rangle$ is the only one having the annihilation property $\tilde{E}_{j i}|1,1 \cdots, 1\rangle=0$ for all $i>j$, therfore it is the so called lowest weight state in the representation theory of Lie algebras. We apply consecutively the different powers of the collective raising operators, $\tilde{E}_{j i}^{\dagger}=\tilde{E}_{i j}=\sum_{n=1}^{N} E_{i j}^{(n)}$, (see definition (2)), and get

$$
\frac{1}{n_{1} ! n_{2} ! \cdots n_{K} !}\left(\frac{N !}{n_{1} ! n_{2} ! \cdots n_{K} !}\right)^{-1 / 2}(\mathbf{1})^{n_{1}} \cdot \tilde{E}_{2,1}^{n_{2}} \cdot \tilde{E}_{3,1}^{n_{3}} \cdots \tilde{E}_{K, 1}^{n_{K}}|1,1, \cdots 1\rangle=\left|n_{1}, n_{2} \ldots n_{K}\right\rangle_{S}
$$

with $\sum_{k=1}^{K} n_{k}=N$. The initial state, as well as all the applied operators according to (2) are symmetric, so the resulting state is also symmetric. Two states of the type $\left|\varphi\left(n_{1}, \ldots n_{K}\right)\right\rangle$ where the series of numbers $n_{1}, \ldots n_{K}$ are not identical are obviously orthogonal, and this property is inherited by their symmetrized versions $\left|n_{1}, n_{2} \ldots n_{K}\right\rangle_{S}$, too. These states are eigenstates of the self-adjoint operators $\tilde{H}_{i 1}$ :

$$
\tilde{H}_{i 1}\left|n_{1}, n_{2} \ldots n_{K}\right\rangle_{S}=\left(n_{i}-n_{1}\right)\left|n_{1}, n_{2} \ldots n_{K}\right\rangle_{S}
$$

therefore a different set of $n_{i}$-s means orthogonal states.

In what follows, it will be expedient to use unnormalized versions of the states $\left|n_{1}, n_{2} \ldots n_{K}\right\rangle_{S}$ :

$$
\left|\begin{array}{c}
N \\
n_{1}, n_{2}, \cdots, n_{K}
\end{array}\right\rangle:=\left(\frac{N !}{n_{1} ! n_{2} ! \cdots n_{K} !}\right)^{1 / 2}\left|n_{1}, n_{2} \ldots n_{K}\right\rangle_{S}
$$

For example: $\left.\left.\cdot\right|_{1,2,0} ^{3}\right\rangle=\sqrt{3}|122\rangle_{S}=|122\rangle+|212\rangle+|221\rangle$ or $\left|\begin{array}{c}4 \\ 3,0,0,1\end{array}\right\rangle=\sqrt{4}|1114\rangle_{S}=|1114\rangle+$ $|1141\rangle+|1411\rangle+|4111\rangle$.

We note that we can get $\left|\begin{array}{c}N \\ n_{1}, n_{2}, \cdots, n_{K}\end{array}\right\rangle$ from the $\left|\varphi\left(n_{1}, \ldots, n_{K}\right)\right\rangle$ states in a direct way. To this end factorise the full permutation group $\mathcal{S}_{N}$ of the $N$ subsystems with the maximum 
stability group of the state $\left|\varphi\left(n_{1}, \ldots n_{K}\right)\right\rangle: \mathcal{S}_{N} / \mathcal{G}$, where $\left.\mathcal{G}=\left\{P_{\nu} \in S_{N}\left|P_{\nu}\right| \varphi\left(n_{1}, \ldots n_{K}\right)\right\rangle=\left|\varphi\left(n_{1}, \ldots n_{K}\right)\right\rangle\right\}$ is the group of all permutations that leave $|\varphi\rangle$ invariant. (The permutations in $\mathcal{G}$ only rearrange those subsystems, which are in the identical states $|k\rangle$ as given by $\left|\varphi\left(n_{1}, \ldots n_{K}\right)\right\rangle$.) Applying the sum of all different representing elements of the coset space we get the unnormalized symmetric states:

$$
\sum_{\tilde{P}_{\nu} \in \mathcal{S}_{N} / \mathcal{G}} \tilde{P}_{\nu}\left|\varphi\left(n_{1}, \ldots, n_{K}\right)\right\rangle=:\left|\begin{array}{c}
N \\
n_{1}, n_{2}, \cdots, n_{K}
\end{array}\right\rangle
$$

\section{$5 \quad$ Generalized coherent states}

We recall the definition of coherent states for a general quantum system [5, 10], applied here for our $N$ quKit states. The construction follows that of the oscillator coherent states, where a continuously parametrized set of unitary displacement operators shifts the ground state to a coherent state. Here we start again with the lowest weight state $|1,1, \cdots 1\rangle$, and apply the unitary displacement operators to it:

$$
\begin{aligned}
|\vec{\eta}\rangle & :=U(\vec{\eta})|1,1 \cdots 1\rangle=\exp \left(\sum_{i>j} \eta_{i j} \tilde{E}_{i j}-\eta_{i j}^{*} \tilde{E}_{i j}^{\dagger}\right)|1,1 \cdots 1\rangle= \\
& =\exp \left(\sum_{n=2}^{K} \eta_{n} \tilde{E}_{n, 1}-\eta_{n}^{*} \tilde{E}_{1, n}\right)|1,1, \cdots, 1\rangle
\end{aligned}
$$

where $\vec{\eta}=\left\{\eta_{i j} \in \mathbb{C}, i>j\right\}$ is an arbitrary set of $K(K-1) / 2$ complex constants. The summation in the expression of $U$ goes only for those operators, which do not annihilate the lowest weight state. The exponential is an antihermitian operator in $\mathbb{S}$, therefore the displacements $U(\vec{\eta})$ are unitary transformations acting in $\mathbb{S}$.

With help of the generalized Baker-Campbell-Haussdorff formula [10] we get:

$$
\begin{aligned}
|\vec{\eta}\rangle & =\exp \left(\sum_{i=2}^{K} \tau_{i} \tilde{E}_{i, 1}\right) \exp \left(\sum_{i=2}^{K} \gamma_{i} \tilde{H}_{i, 1}\right) \exp \left(-\sum_{i=2}^{K} \tau_{i} \tilde{E}_{1, i}\right)|1,1 \cdots 1\rangle= \\
& =\mathcal{N} \exp \left(\sum_{i=2}^{K} \tau_{i} \tilde{E}_{i, 1}\right)|1,1 \cdots 1\rangle
\end{aligned}
$$

where $\mathcal{N}$ is an appropriate normalization factor and $\tau_{i} \in \mathbb{C}$ are some complex constants.

This follows from $\left[\tilde{E}_{i, 1}, \tilde{E}_{1, j}\right]=\tilde{E}_{i, j}$ for $i \neq j$, and from the facts that $|1,1 \cdots 1\rangle$ is the eigenstate of $\left[\tilde{E}_{i, 1}, \tilde{E}_{1, i}\right]=\tilde{H}_{i, 1}$ and that all $\tilde{E}_{1, i^{-}}$s annihilate $|1,1 \cdots 1\rangle$. 


\section{The coherent states are product states, and these are the only ones}

Using (19) we can show, that the states $|\vec{\eta}\rangle$ can be factorized into products of quKits, as follows

$$
\begin{aligned}
|\vec{\eta}\rangle & =\mathcal{N} \exp \left(\sum_{i=2}^{K} \tau_{i} \tilde{E}_{i, 1}\right)|1,1 \cdots 1\rangle=\mathcal{N} \exp \left(\sum_{i=2}^{K} \tau_{i} \sum_{n=1}^{N} E_{i, 1}^{(n)}\right)|1\rangle_{1} \otimes|1\rangle_{2} \otimes \cdots \otimes|1\rangle_{N}= \\
& =\mathcal{N} \exp \left(\sum_{i=2}^{K} \tau_{i} E_{i, 1}^{(1)}\right)|1\rangle_{1} \otimes \exp \left(\sum_{i=2}^{K} \tau_{i} E_{i, 1}^{(2)}\right)|1\rangle_{2} \otimes \cdots \otimes \exp \left(\sum_{i=2}^{K} \tau_{i} E_{i, 1}^{(N)}\right)|1\rangle_{N}= \\
& =\mathcal{N} \bigotimes_{n=1}^{N} \exp \left(\sum_{i=2}^{K} \tau_{i} E_{i, 1}^{(n)}\right)|1\rangle_{n}=\mathcal{N} \bigotimes_{n=1}^{N}\left(1+\sum_{i=2}^{K} \tau_{i} E_{i, 1}^{(n)}\right)|1\rangle_{n}= \\
& =\mathcal{N} \bigotimes_{n=1}^{N}\left(|1\rangle+\tau_{2}|2\rangle+\tau_{3}|3\rangle+\cdots+\tau_{K}|K\rangle\right)_{n}
\end{aligned}
$$

Here we have used that

$$
\exp \left(\sum_{i=2}^{K} \tau_{i} E_{i, 1}^{(n)}\right)|1\rangle_{n}=\sum_{q=0}^{\infty} \frac{1}{q !}\left(\sum_{i=2}^{K} \tau_{i} E_{i, 1}^{(n)}\right)^{q}|1\rangle_{n}=\left(\mathbf{1}+\sum_{i=2}^{K} \tau_{i} E_{i, 1}^{(n)}\right)|1\rangle_{n}
$$

and exploited that $E_{i, 1}^{(n)}$-s "moves" only the $|1\rangle$ basis state, so $\left(E_{j, 1}\right)^{q_{j}}\left(E_{i, 1}\right)^{q_{i}}|1\rangle_{n}=0$ for all $i$ and $j$ if $q_{i}+q_{j} \geqq 2$.

Now we prove that in the symmetric subspace the notentangled states are exactly the coherent states. We can write any pure state in $\mathbb{S}$ as a linear combination of the unnormalized states introduced in (16):

$$
\begin{aligned}
|\psi\rangle & =\sum_{n_{1}+\cdots+n_{K}=N} C_{n_{1}, n_{2}, \cdots, n_{K}}\left|\begin{array}{c}
N \\
n_{1}, n_{2}, \cdots, n_{K}
\end{array}\right\rangle= \\
& =\sum_{i_{2}=0}^{N} \sum_{i_{3}=0}^{N} \cdots \sum_{i_{K}=0}^{N} C_{N-i_{2}-i_{3}-\cdots-i_{K}, i_{2}, \cdots, i_{K}}\left|\begin{array}{c}
N-i_{2}-i_{3}-\cdots-i_{K}, i_{2}, \cdots, i_{K}
\end{array}\right\rangle .
\end{aligned}
$$

$\langle\psi \mid \psi\rangle=1$ then requires:

$$
\sum_{n_{1}+\cdots+n_{K}=N}^{N}\left|C_{n_{1}, n_{2}, \cdots, n_{K}}\right|^{2} \frac{N !}{n_{1} ! n_{2} ! \cdots n_{K} !}=1
$$


The states $\left|\begin{array}{c}N \\ n_{1}, n_{2}, \cdots, n_{K}\end{array}\right\rangle$-s have the following property

$$
\begin{gathered}
\left|\begin{array}{c}
N \\
n_{1}, n_{2}, \cdots, n_{K}
\end{array}\right\rangle=|1\rangle_{n} \otimes\left|\begin{array}{c}
N-1 \\
n_{1}-1, n_{2}, \cdots, n_{K}
\end{array}\right\rangle+|2\rangle_{n} \otimes\left|\begin{array}{c}
N-1 \\
n_{1}, n_{2}-1, \cdots, n_{K}
\end{array}\right\rangle+\cdots \\
\cdots+|K\rangle_{n} \otimes\left|\begin{array}{c}
N-1 \\
n_{1}, n_{2}, \cdots, n_{K}-1
\end{array}\right\rangle=\sum_{k=1}^{N-1}|k\rangle_{n} \otimes\left|\begin{array}{c}
N \\
n_{1}, n_{2}, \cdots, n_{k}-1, \cdots, n_{K}
\end{array}\right\rangle,
\end{gathered}
$$

where $\left|\begin{array}{c}N \\ n_{1}, n_{2}, \cdots, n_{K}\end{array}\right\rangle=0$ by definition, if $n_{k}>N$ or $n_{k}<0$ for $k \in\{1,2, \cdots, K\}$. The above decompositions, which correspond to the elementary combinatoric identity $\frac{N !}{n_{1} ! n_{2} ! \cdots n_{K} !}=$ $\sum_{k=1}^{K} \frac{(N-1) !}{n_{1} ! \cdots\left(n_{k}-1\right) ! \cdots n_{K} !}$ are valid for any $n=1, \ldots N$, as a consequence of the symmetry of the states $\left|\begin{array}{c}N \\ n_{1}, n_{2}, \cdots, n_{K}\end{array}\right\rangle$ with respect of permutations.

Then using (24), we get:

$$
\begin{aligned}
|\psi\rangle & =\sum_{n_{1}+\cdots+n_{K}=N} C_{n_{1}, n_{2}, \cdots, n_{K}}\left|\begin{array}{c}
N \\
n_{1}, n_{2}, \cdots, n_{K}
\end{array}\right\rangle \\
& \left.=\left.\sum_{n_{1}+\cdots+n_{K}=N} C_{n_{1}, n_{2}, \cdots, n_{K}} \sum_{k=1}^{K}|k\rangle_{n} \otimes\right|_{n_{1}, n_{2}, \cdots, n_{k}-1, \cdots, n_{K}}\right\rangle= \\
& =\sum_{i=1}^{K}\left(|k\rangle_{n} \otimes \sum_{n_{1}+\cdots+n_{K}=N} C_{n_{1}, n_{2}, \cdots, n_{K}}\left|\begin{array}{c}
N-1 \\
n_{1}, n_{2}, \cdots, n_{k}-1, \cdots, n_{K}
\end{array}\right\rangle\right) .
\end{aligned}
$$

As $|\psi\rangle$ is an arbitrary not entangled state, so according to the parallellity criterion of section 3, the following $N-1$ partite states should be parallel to each other:

$$
\begin{gathered}
\sum_{n_{1}+\cdots+n_{K}=N} C_{n_{1}, n_{2}, \cdots, n_{K}}\left|\begin{array}{c}
N-1 \\
n_{1}-1, n_{2}, \cdots, n_{K}
\end{array}\right\rangle \\
\sum_{n_{1}+\cdots+n_{K}=N} C_{n_{1}, n_{2}, \cdots, n_{K}}\left|\begin{array}{c}
N-1 \\
n_{1}, n_{2}-1, \cdots, n_{K}
\end{array}\right\rangle= \\
\sum_{n_{1}+\cdots+n_{K}=N} C_{n_{1}, n_{2}, \cdots, n_{K}}\left|\begin{array}{c}
N+n_{K}=N \\
n_{1}, n_{2}, \cdots, n_{K}-1
\end{array}\right\rangle= \\
\sum_{n_{1}-1, n_{2}+1, \cdots, n_{K}}\left|\begin{array}{c}
N-1 \\
n_{1}-1, n_{2}, \cdots, n_{K}
\end{array}\right\rangle \\
\sum_{n_{1}+\cdots+n_{K}=N} C_{n_{1}-1, n_{2}, \cdots, n_{K}+1}\left|\begin{array}{c}
N-1 \\
n_{1}-1, n_{2}, \cdots, n_{K}
\end{array}\right\rangle
\end{gathered}
$$

By changing the summation indices in the right hand sides as $n_{1} \rightarrow n_{1}^{\prime}=n_{1}+1 ; n_{k} \rightarrow n_{k}^{\prime}=$ $n_{k}-1$, we see that the parallellity of these states requires :

$$
\begin{gathered}
\tau_{2} \cdot C_{n_{1}, n_{2}, \cdots, n_{K}}=C_{n_{1}-1, n_{2}+1, \cdots, n_{K}} \\
\vdots \\
\tau_{K} \cdot C_{n_{1}, n_{2}, \cdots, n_{K}}=C_{n_{1}-1, n_{2}, \cdots, n_{K}+1}
\end{gathered}
$$


where $\tau_{i}(i \in\{2, \cdots, K\})$.are arbitrary complex numbers.

This means that $C_{N-i_{2}-i_{3}-\cdots-i_{K}, i_{2}, \cdots, i_{K}}$ must be of the form:

$$
C_{N-i_{2}-i_{3}-\cdots-i_{K}, i_{2}, \cdots, i_{K}}=\tau_{2}^{i_{2}} \cdots \cdots \tau_{K}^{i_{K}} \cdot C_{N, 0, \cdots, 0}
$$

And thus - comparing with (20) - we immediately see that $|\psi\rangle$ is a coherent state

$$
\begin{gathered}
|\psi\rangle=\sum_{i_{2}=0}^{N} \sum_{i_{3}=0}^{N} \cdots \sum_{i_{K}=0}^{N} C_{N-i_{2}-i_{3}-\cdots-i_{K}, i_{2}, \cdots, i_{K}}\left|\begin{array}{c}
N \\
N-i_{2}-i_{3}-\cdots-i_{K}, i_{2}, \cdots, i_{K}
\end{array}\right\rangle \\
=\sum_{i_{2}=0}^{N} \sum_{i_{3}=0}^{N} \cdots \sum_{i_{K}=0}^{N} \tau_{2}^{i_{2}} \cdots \cdots \tau_{K}^{i_{K}} \cdot C_{N, 0, \cdots, 0}\left|\begin{array}{c}
N \\
N-i_{2}-i_{3}-\cdots-i_{K}, i_{2}, \cdots, i_{K}
\end{array}\right\rangle \\
=C_{N, 0, \cdots, 0} \cdot\left(|1\rangle+\tau_{2}|2\rangle+\cdots+\tau_{K}|K\rangle\right)^{\otimes N}
\end{gathered}
$$

\section{The vectors orthogonal to the symmetric subspace are all entangled}

We shall now consider vectors in $\mathbb{S}_{\perp}$ the orthogonal complement of the symmetric space. We prove that all vectors in $\mathbb{S}_{\perp}$ are globally entangled. To this end, assume to the contrary, that there exists a vector $|\varphi\rangle \in \mathbb{S}_{\perp}$ which can be written as a product:

$$
|\varphi\rangle=\bigotimes_{n=1}^{N}\left(a_{n}^{1}|1\rangle_{n}+a_{n}^{2}|2\rangle_{n}+\ldots a_{n}^{K}|K\rangle_{n}\right)=\bigotimes_{n=1}^{N} \sum_{k=1}^{K} a_{n}^{k}|k\rangle_{n}
$$

with $\sum_{k=1}^{K}\left|a_{n}^{k}\right|^{2}=1$ for each $n=1 . \cdots N$.

As $|\varphi\rangle \in \mathbb{S}_{\perp}$ it cannot have a nonzero projection onto an arbitrary symmetric state.

Consider first the projection of $|\varphi\rangle$ onto the symmetric state: $\left|\begin{array}{c}N, 0, \cdots, 0 \\ N\end{array}\right\rangle=|1\rangle_{1} \otimes|1\rangle_{2} \otimes$ $\cdots \otimes|1\rangle_{N}$. This must be zero, which demands $\prod_{n=1}^{N} a_{n}^{1}=0$, so at least one of the $a_{n}^{1}$-s must be zero. Without loss of generality we may assume that the vanishing coefficient is $a_{1}^{1}=0$.

Then $|\varphi\rangle=\left(a_{1}^{2}|2\rangle+a_{1}^{3}|3\rangle+\cdots+a_{1}^{K}|K\rangle\right)_{1} \bigotimes_{n=2}^{N}\left(a_{n}^{1}|1\rangle+a_{n}^{2}|2\rangle+a_{n}^{3}|3\rangle+\cdots+a_{n}^{K}|K\rangle\right)_{n}$

Consider now the projections onto the symmetric states with $n_{1}=N-1$, which are $\left.\left.\frac{1}{\sqrt{N}}\left|\begin{array}{c}N \\ N-1,1, \cdots, 0\end{array}\right\rangle ;\left.\frac{1}{\sqrt{N}}\right|_{N-1,0,1,0 \cdots, 0} ^{N}\right\rangle ;\left.\cdots \cdots \frac{1}{\sqrt{N}}\right|_{N-1,0, \cdots, 1} ^{N}\right\rangle$. As these projections must be zero again, we have $a_{1}^{k} \prod_{n=2}^{N} a_{n}^{1}=0$, for all possible $k=2, \cdots, K$. All the $a_{1}^{k}$-s can not be zero, because then $|\varphi\rangle$ would be zero, so $\prod_{n=2}^{N} a_{n}^{1}=0$ and again at least one of the $a_{n}^{1}$-s $(n \neq 1)$ must be zero. Without loss of generality we may assume that the vanishing coefficient is $a_{2}^{1}=0$. 
Next we consider the symmetric states with $n_{1}=N-2$. The projections onto these states again must be zero, which leads us finally to $\prod_{n=3}^{N} a_{n}^{1}=0$. Again one must be zero, say $a_{3 .}^{1}$. Continuing in this way with $n_{1}=N-3, \cdots, 1,0$ we finally arrive to $a_{1}^{1}=a_{2}^{1}=\cdots=a_{N}^{1}=0$.

If we carry out the same reasoning starting with the state corresponding to $n_{2}=N$, we get $a_{1}^{2}=a_{2}^{2}=\cdots=a_{N}^{2}=0$, and continuing in this way, with $n_{3}=\cdots=n_{K}=N$, we get at the end that all the $a_{n}^{k}$-s are zero, so $|\varphi\rangle=0$.

We arrived to a contradiction: the nonentangled $|\varphi\rangle$ cannot be orthogonal to $\mathbb{S}$, or stated otherwise: all elements of $\mathbb{S}_{\perp}$ are entangled.

In conclusion, our result shows that besides of other characteristics showing quasiclassicity of coherent states, there exists an additional remarkable one: they are the only nonentangled pure states in a symmetric multipartite system of quKits. Moreover, all states in $\mathbb{S}_{\perp}$, in the orthogonal complement of the symmetric subspace, are entangled. We note, however, that the analogy between oscillator coherent states and the ones considered here cannot be extended to the question of entanglement, as an oscillator mode is a single system, while atomic coherent states are multipartite by their definition, which is a necessary condition of entanglement.

The work was supported by the Hungarian Scientific Research Fund (OTKA) under contract No: T48888. We thank L. Fehér for useful discussions.

\section{References}

[1] M. A. Nielsen, I. L. Chuang, Quantum Computation and Quantum Information (Cambridge University Press, Cambridge, 2000).

[2] P. Horodecki, R. Horodecki, M Horodecki, K. Horodecki, arXiv:quant-ph/0702225v2.

[3] R. J. Glauber, Phys. Rev. 131 (1963) 2766.

[4] J. R. Klauder and B. S. Skagerstam 1985 Coherent States - Applications in Physics and Mathematical Physics (Singapore: World Scientific)

[5] A.M. Perelomov, Generalized Coherent States and their Applications (Berlin Springer, 1986)

[6] S. T. Ali, J. P. Antoine and J. P. Gazeau 2000 Coherent States, Wavelets and Their Generalizations (Berlin: Springer)

[7] W. H. Zurek, S. Habib and J. P. Paz 1993 Phys. Rev. Lett. 701187.

[8] P. Földi, A. Czirják and M. G. Benedict 2001 Phys. Rev. A. 6333807

[9] F. Arecchi, E. Courtens, R. Gilmore, H. Thomas, Phys. Rev. A 6 (1972) 2211.

[10] W. M. Zhang, D. H. Feng, R. Gilmore, Rev. Mod. Phys. 62 (1990) 867 
[11] P. Dömötör and M. G. Benedict 2008 Phys. Lett. A 372, 3792-3795

[12] C. Brif, A. Mann and M. Revzen 1998 Phys. Rev. A 57 742. Note that the use of the term Bell state in this reference is not how we use it today: their Bell states are unentangled

[13] T. Ichikawa, T. Sasaki, I. Tsutsui and N. Yonezawa arXiv:0805.3625

[14] R. Gilmore 1974 Lie Groups Lie Algebras and Some of their Applications (New York: Wiley)

[15] D. A. Meyer and N. R. Wallach 2002 J. Math. Phys. 43 